Prpic et al.

\title{
Octave bias in pitch perception: The influence of pitch height
}

\section{on pitch class identification}

Valter Prpic ${ }^{1}$, Mauro Murgia ${ }^{1}$, Matteo De Tommaso ${ }^{2}$, Giulia Boschetti ${ }^{1}$, Alessandra Galmonte ${ }^{3}$ and Tiziano Agostini ${ }^{1}$

${ }^{1}$ Department of Life Sciences, University of Trieste, Italy

${ }^{2}$ Center for Mind/Brain Sciences, University of Trento, Italy

${ }^{3}$ Department of Neurological, Biomedical and Movement Sciences, University of Verona, Italy

Accepted for publication in Perception

March 21st 2016 
Prpic et al.

\section{Corresponding author}

Valter Prpic, Department of Life Sciences, University of Trieste,

via Weiss, 21 - building "W" - 34128 Trieste, Italy.

Email Address: valter.prpic@gmail.com 
Prpic et al.

\section{Acknowledgments}

A special thanks goes to the State Conservatory of Music G. Tartini, Trieste (Italy), for letting us test their students and use the conservatory facilities. We thank Corinna Pannofino for the English proofreading. 


\section{Prpic et al.}

\section{Abstract}

Pitch height and pitch class are different, but strictly related, percepts of music tones. To investigate the influence of pitch height in a pitch class identification task, we systematically analyzed the errors in-terms of direction and amount - committed by a group of musicians. The aim of our study was to verify the existence of constant errors in the identification of pitch classes across consecutive octaves. Stimuli were single piano tones from the $\mathrm{C}$ major scale executed in two consecutive octaves. Participants showed different response patterns in the two octaves. The direction of errors revealed a constant tendency to underestimate pitch classes in the lowest octave and to overestimate pitch classes in the highest octave. Thus, pitch height showed to influence pitch class identification. We called this bias "pitch class polarization", since the same pitch class was judged to be respectively lower and higher, depending on relatively low/high pitch height.

\section{Keywords}

Pitch class, pitch height, octave equivalence, octave bias, polarization. 
Prpic et al.

\section{Introduction}

In the twelve-tone equal temperament, musical tones are categorized in 12 pitch classes (C, C\#, D, D\#, E, F, F\#, G, G\#, A, A\# and B). These pitch classes divide each octave, by semitone distances, into an equivalent number of intervals. Tones which are one semitone apart from each other (e.g., C-C\#, E-F) have fundamental frequencies forming a $2^{1 / 12}: 1$ ratio. Instead, an octave is defined as the interval between two pitches that have half or double frequency (1:2 ratio). Two pitches separated by an octave belong to the same pitch class and, thus, have the same letter name. Commonly, the octave designation is indicated by a number following the pitch class letter (e.g., $\mathrm{C}_{4}$ ). Therefore, the middle $\mathrm{C}$ $(261.6 \mathrm{~Hz})$ is designated as $\mathrm{C}_{4}$, while the $\mathrm{C}(523.2 \mathrm{~Hz})$ above middle $\mathrm{C}$ is $\mathrm{C}_{5}$ and the $\mathrm{C}(130.8 \mathrm{~Hz})$ below middle $\mathrm{C}$ is $\mathrm{C}_{3}$ (Owen, 2000).

In music theory, according to octave equivalence, tones separated by one or more octaves are considered almost musically identical (Babbitt, 1960; 1965; Forte, 1973). Indeed, all cultures consider tones which are one octave apart as similar (Burns \& Ward, 1978; Crickmore, 2003). In twelve-tone equal temperament, equivalent tones share the same pitch class designation (e.g., the pitch class $C$ is labelled in the same way across all octaves), but they differ in terms of pitch height. Pitch height refers to the absolute frequency of a tone (i.e., the more two tones differ in frequency, the more they differ in pitch height). Pitch height and pitch class are different percepts. Indeed, two tones separated by one semitone are similar in pitch height, but they belong to different pitch classes; whereas two tones separated by one octave are different in pitch height, but equal in terms of pitch class (Shepard, 1982; Oxenham, 2013). The pitch helix is a simple and effective spatial model for representing the relationship between pitch class and pitch height. All twelve pitch classes are represented around the helix on the horizontal plane whereas pitch height is represented on the vertical plane, thus the 


\section{Prpic et al.}

resulting helix is the combination of these two planes (Figure 1). Empirical evidence supports this representational model (Deutsch, Dooley \& Henthorn, 2008), suggesting that musical pitch varies along these two dimensions (i.e., the monotonic dimension of pitch height and the circular dimension of pitch class).

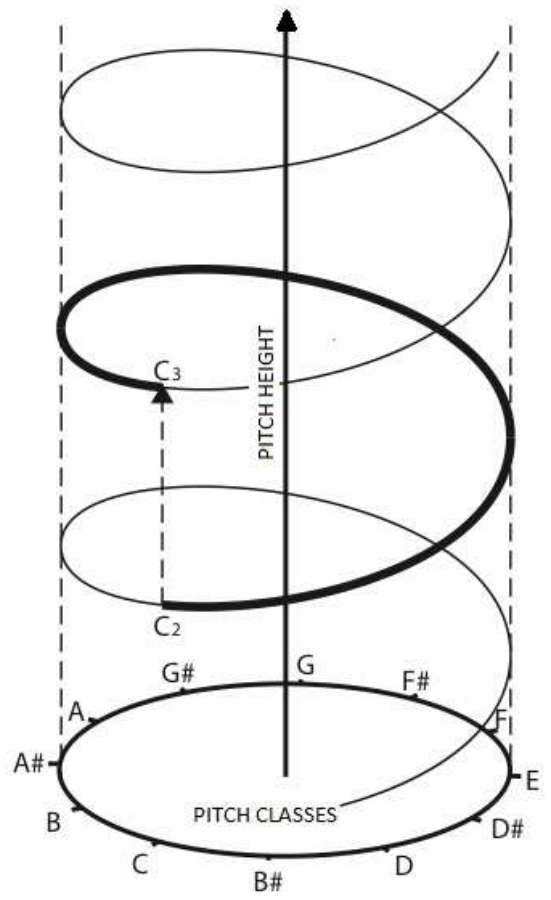

Figure 1. Representation of the pitch helix. Modified from Shepard (1982).

Although the concept of octave equivalence is well defined in music theory, empirical evidence suggests that this phenomenon is related to individuals' experience (for a review, see Burns, 1999). Overall, experiments that required adult humans to judge the similarity of isolated pairs of pure tones 


\section{Prpic et al.}

showed no evidence at all, or a very weak effect of similarity between the octaves in non-musicians (Allen, 1967; Kallman, 1982; Umemoto, 1990; Thurlow \& Erchul, 1977). Conversely, most musicians in these studies showed a clear effect of octave equivalence.

Incongruent evidence with the theoretical concept of octave equivalence was revealed by non-experts also in the identification of simple melodies. Indeed, the alteration of the octaves of several tones showed to effectively impair the identification of melodies (Deutsch, 1972). Conversely, using go/no-go operant discrimination and generalization, Hoeschele, Weisman, \& Sturdy (2012) have recently provided new evidence in favour of octave equivalence in adult humans, regardless of music training. Overall, studies on octave equivalence struggled to show clear empirical evidence of this music phenomenon in non-experts. However, it seems quite clear that people with musical experience effectively perceive octave equivalence.

Octave equivalence is a basic assumption for the identification of pitch classes. The pitch class identification task has traditionally been used to study the ability of a peculiar group of individuals, namely, the absolute pitch (AP) possessors. Standing to a wide and well-known literature, this ability allows individuals to associate a target pitch class to its label, without requiring particular strategies or effort (Ward \& Burns, 1999; Levitin \& Rogers, 2005; Takeuchi \& Hulse, 1993; Vanzella \& Schellenberg, 2010; Wynn, 1993). However, many authors consider the AP ability as a rare $(0.01 \%$ of the general population according to Bachem, 1955; Profita \& Bidder, 1988) and controversial phenomenon (see Deutsch, 2013).

Although AP possessors have the exceptional ability to identify pitch class, both AP and non-AP possessors showed to be considerably affected by pitch height of stimuli in their identification abilities. Indeed, several studies reported that identifications in central octaves were faster (Baird, 1917) and more accurate (Bachem, 1937; Miyazaki, 1989; Rakowski \& Morawska-Bungeler, 1987; Ward, 1963) 


\section{Prpic et al.}

compared to those in extremely high or low octaves. Similarly, the sensitivity to tonality revealed to be dramatically and moderately reduced in the low and high pitch register, respectively (Russo, Cuddy, Galembo \& Thompson, 2007). Although AP possessors are more accurate in pitch class identification, most studies revealed no differences in octave identification between AP and non AP musicians (Bachem, 1955; Baird, 1917; Lockhead \& Byrd, 1981; Miyazaki, 1989, Rakowski \& Morawska-Bungeler, 1987; Weisman, Balkwill, Hoeschele, Moscicki, Bloomfield, \& Sturdy, 2010). Furthermore, a great majority of identification errors committed by AP possessors can be ascribed to octave errors (Bachem, 1937; Baird, 1917; Lockhead \& Byrd, 1981; Miyazaki, 1989). Therefore, some studies did not consider octave errors as errors during pitch class identification tasks (Zatorre \& Beckett, 1989), or directly required participants to identify the pitch class without considering the octave (Miyazaki, 1989). Taken together, this evidence confirms the conceptual difference between pitch class and pitch height. However, it also shows how these features of a tone interact in human perception.

In addition to pitch height, several studies showed that the accuracy of pitch class identifications can vary as a function of instrument timbre and it is not equal for all pitch classes. Among all musical instruments and electronically produced timbres (e.g., pure tones), pitches with piano timbre seem to be the easiest to identify (Bachem, 1937; Baird, 1917). Furthermore, a great familiarity with a timbre can make pitch identifications easier (Brammer, 1951). The accuracy and the speed of pitch class identification depend also on the specific pitch classes. Indeed, "white-key pitches", namely pitches that do not have sharp or flat in the pitch class name and correspond to the white keys of the piano, are identified more accurately than "black-key pitches" (Baird, 1917; Miyazaki, 1989; Takeuchi \& Hulse, 1991).

From a methodological perspective, our review of literature revealed that the influence of pitch height in pitch class identification has been mostly studied in terms of accuracy. To the best of our knowledge, 8 


\section{Prpic et al.}

no study has systematically analyzed the errors committed by participants, in terms of direction and amount. The study of this aspect is particularly relevant because it would allow us to understand whether or not pitch class identification is influenced by systematic biases, due to pitch height.

To address this issue, we decided to test pianists with formal music education, using a pitch class identification task. We decided not to include AP possessors because they would commit just a slight number of errors and, thus, they would not provide useful information for our level of analyses. To facilitate participants' tasks we chose a very familiar set of stimuli, which were relatively easy to identify (i.e., white-key piano tones recorded in the two central octaves). The aim of our work was to verify the existence of constant errors in the identification of pitch classes across consecutive octaves. Given that the pitch height has a reliable influence on the accuracy of pitch class identification, we aimed to investigate the direction and the amount of these errors, measured in semitones and whole tones.

\section{Method}

\section{Participants}

Twenty-one advanced students or recently graduated musicians from the State Conservatory of Music "G. Tartini" (Italy) took part in the experiment. They were 11 males and 10 females (mean age 23.9; SD $=2.6)$ with a mean of $7.3(S D=2.5)$ years of formal studies in piano playing. All participants selfreported that they were not AP possessors. They all provided their informed consent prior to inclusion in the study and were unaware of the aims of the research. 


\section{Prpic et al.}

\section{Apparatus and stimuli}

The presentation of the stimuli was programmed and controlled by the E-Prime 2.0 software, running on a Dell notebook (Intel Core 13, 2 GB RAM). Stimuli were 14 piano tones with a duration of 3000 ms each, previously recorded with a professional Kurweil PC3 LE8 digital piano, and consisted in the C major scale repeated in the 3rd and 4th octave. On the music staff, notes in the 3rd octave are represented in the bass clef, while notes in the 4th octave are represented in the treble clef. Participants listened to the stimuli through professional headphones (AKG K121). The volume was set on a comfortable fixed level (about $65 \mathrm{~dB}$ ) for all the participants. Responses were recorded either with an electric piano keyboard (Casio PX350 BK) or with a voice microphone (Shure PG58). Both response devices were connected to an external audio device (M-Audio Mobile Pre USB) connected to a MacBook Pro (Intel Core i5, 4 GB RAM). The experiment took place in a quiet room of the State Conservatory of Music "G. Tartini", without environmental distractions.

\section{Procedure}

All the participants were required to identify the pitch class of the stimuli. Participants were told to ignore the octave of the pitch and to respond exclusively by identifying the pitch class. Participants were informed that all the stimuli were pitches of the $C$ major scale, however, they were not aware that the stimuli belonged only to the third and fourth octaves. Moreover, they were not given any pitch reference before or during the task.

The stimuli were presented one-by-one for $3000 \mathrm{~ms}$ and were followed by $2000 \mathrm{~ms}$ of silence. Participants could respond any time after each stimulus had started, so they had globally $5000 \mathrm{~ms}$ to respond. Responses after 5000 ms were not accepted. After this time interval, before a new trial, a distracting drum sequence of 2000 ms started. This sequence was a fast drum rhythm played on bass 


\section{Prpic et al.}

drum, snare drum and cymbals, in order to provide the listener with a wider range of frequencies (low, medium, high). The aim of this distracting sound sequence was to interfere with participants' echoic memory and, therefore, to discourage participants from using previous stimuli as references for next trials. Indeed, we meant to investigate participants' errors in absolute pitch judgments, thus we had to prevent the retention in memory of previous stimuli (for additional information about memory for pitches and other musical attributes, see Levitin, 2002). Both response times and accuracy were stressed in the instructions.

Participants' responses were obtained in two conditions (motor or verbal response). In the motor response condition, participants were required to press the correct key of the electric piano corresponding to the listened pitch class. The electric piano was muted, thus no external pitch reference was provided to participants during the identification task. In the verbal response condition, they were required to identify the target pitch by naming its pitch class, by using Italian note names (e.g. DO, RE, MI).

The order of the conditions was counterbalanced, thus half of participants started with the verbal condition, while the other half started with the motor one. Each condition was set up by 5 repetitions of the entire stimulus set ( 7 notes $\times 2$ octaves), resulting in a total of 70 experimental trials for each condition. All 70 stimuli of each condition were presented in random order, but a stimulus could not be presented twice consecutively. Participants had the possibility to take a short break after completing the first condition. To practice with the task, each condition was preceded by 14 practice trials in random order (the complete stimulus set) which were excluded from data analyses.

For data analyses, we calculated the direction and the amount of errors in pitch class identification (ignoring pitch height) as follows: correct responses were considered 0 and, therefore, they were not taken into account; errors could be either positive (+) or negative (-). We considered overestimation of 


\section{Prpic et al.}

pitch classes (e.g., if the target tone was $\mathrm{A}$ and a participant responded $\mathrm{B}, \mathrm{C}$ or $\mathrm{D}$ ) as positive errors, while underestimation of pitch classes (e.g., if the target tone was $A$ and a participant responded G, F or E) were considered as negative errors. Moreover, we considered the error amount by calculating the "Interval Error", namely, the distance in tones ( 1 unit) and semitones ( 0.5 unit) between the participants' response and the actual target pitch class (Table 1). The errors were always calculated on the closest note. For instance, if a participant responded B on a target $\mathrm{C} 4$, this was considered as a B3 and not a B4, thus it was coded as a deviation of -0.5 semitone and not as a deviation of +11 semitones. A similar approach to pitch errors was previously used by Levitin (1994) for analyzing participants' errors during a singing production task. Finally, for each target tone, we separately calculated the sum of the interval errors for both octaves. Therefore, positive values $(+)$ indicated a tendency to overestimate pitch classes, while negative values (-) indicated a tendency to underestimate pitch classes. It is noteworthy that both octaves contain the same notes and that the expected value for errors across all notes is 0 .

\begin{tabular}{llllllll}
\hline & C & D & E & $F$ & $G$ & $A$ & $B$ \\
\hline$C$ & 0.0 & 1.0 & 2.0 & 2.5 & -2.5 & -1.5 & -0.5 \\
\hline $\mathrm{D}$ & -1.0 & 0.0 & 1.0 & 1.5 & 2.5 & -2.5 & -1.5 \\
\hline $\mathrm{E}$ & -2.0 & -1.0 & 0.0 & 0.5 & 1.5 & 2.5 & -2.5 \\
\hline $\mathrm{F}$ & -2.5 & -1.5 & -0.5 & 0.0 & 1.0 & 2.0 & 3.0 \\
\hline & & & & & & & \\
\hline
\end{tabular}


Prpic et al.

\begin{tabular}{llllllll}
\hline G & 2.5 & -2.5 & -1.5 & -1.0 & 0.0 & 1.0 & 2.0 \\
\hline A & 1.5 & 2.5 & -2.5 & -2.0 & -1.0 & 0.0 & 1.0 \\
\hline B & 0.5 & 1.5 & 2.5 & -3.0 & -2.0 & -1.0 & 0.0 \\
\hline
\end{tabular}

Table 1. Coding of Interval Errors, depending on the congruency between the pitch class of the stimuli (rows) and participants' responses (columns). For instance, the error of the response " $G$ " for a stimulus "F" was coded as +1 (two semitone error); while a response " $E$ " was coded as -0.5 (one semitone error).

\section{Data analysis and results}

A repeated-measures ANOVA was run for the Interval Errors, with a $2 \times 2 \times 7$ design (Response Condition $x$ Octave $x$ Pitch Class). The results revealed a significant main effect of Octave $[F(1,20)=6.35 ; p<.05$; $\left.\eta^{2}=.24\right]$, indicating a difference in the interval errors committed in the relatively low $(M=-0.61$ tones) and high ( $M=0.76$ tones) octaves (Figure 2$)$. Conversely, both the main effect of response condition $[F$ $(1,20)=0.04 ; p=0.85]$ as well as the main effect of the pitch class $[F(6,120)=0.37 ; p=0.90]$ were not significant. 
Prpic et al.

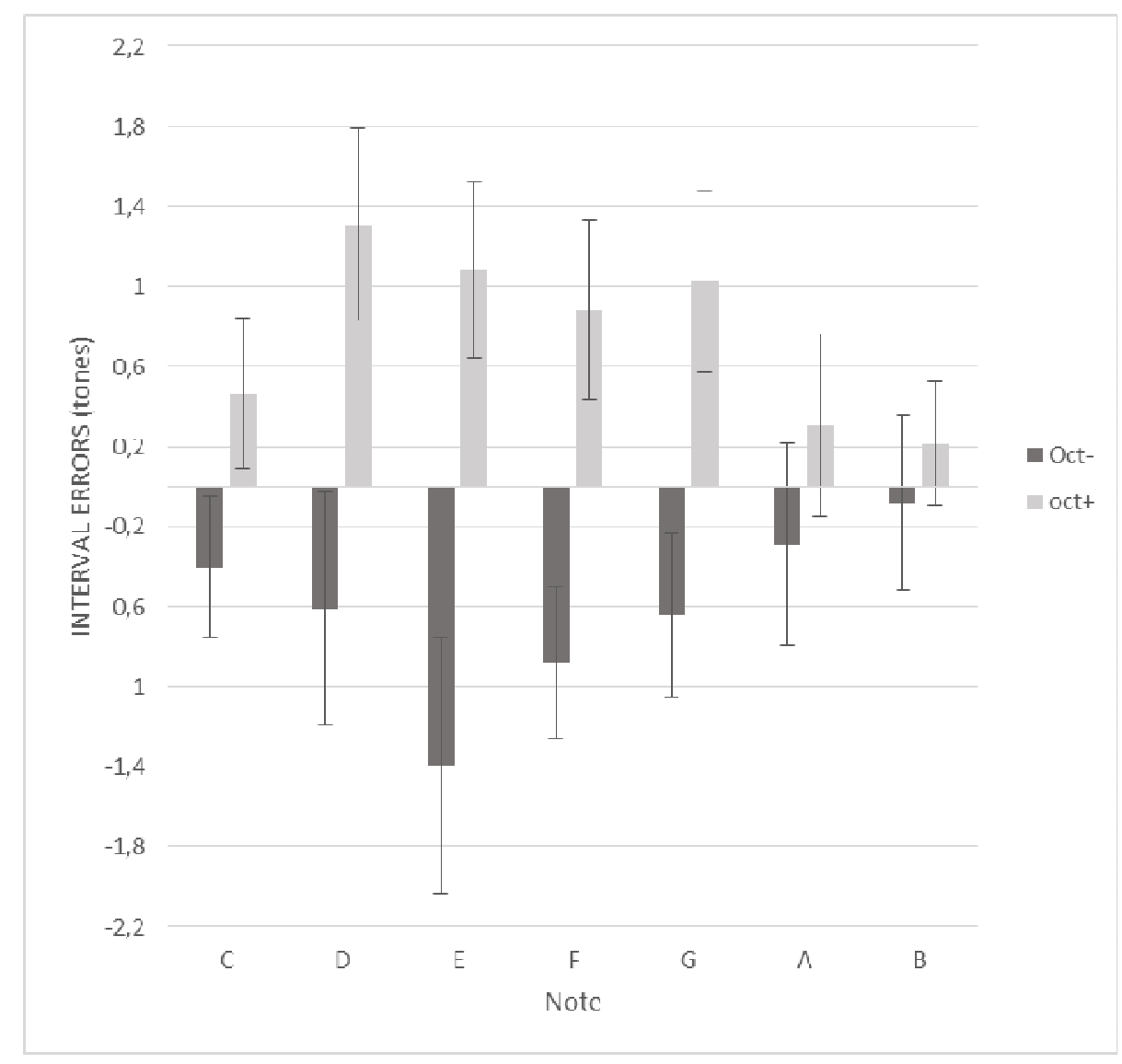

Figure 2. Results for interval errors. Dark gray lines represent the lower octave and light gray lines the higher octave. Error bars represent standard errors of the mean.

Moreover, the results revealed significant values for the interactions Response Condition $\mathrm{x}$ Octave $\mathrm{x}$ Pitch Class, $\left[F(6,120)=2.71 ; p<.05 ; \eta^{2}=.12\right]$, and Octave $x$ Pitch Class, $\left[F(6,120)=2.31 ; p<.05 ; \eta^{2}=\right.$ .10]; while the interaction Response Condition $x$ Octave was not significant, $[F(1,20)=0.17 ; p=.68]$. Additional within subjects $t$-tests were run to investigate differences across the two octaves for the single pitch classes. The results revealed significant differences across the two octaves for 3 pitch classes, namely, $D[t(20)=-2.22 ; p<.05 ; d=.56], E[t(20)=-2.73 ; p<.05 ; d=.65]$, and $F[t(20)=-2.70$; 


\section{Prpic et al.}

$p<.05 ; d=.73]$, and a marginally significant result for $G[t(20)=-2.07, p=0.052 ; d=.30]$. The frequencies of participants responses for each stimulus are reported in Table 2.

\begin{tabular}{ccccccccc}
\hline & $\mathrm{C}$ & $\mathrm{D}$ & $\mathrm{E}$ & $\mathrm{F}$ & $\mathrm{G}$ & $\mathrm{A}$ & $\mathrm{B}$ & $\begin{array}{c}\text { No } \\
\text { response }\end{array}$ \\
\hline $\mathrm{C}_{3}$ & 172 & 6 & 3 & 3 & 8 & 9 & 6 & 3 \\
\hline $\mathrm{D}_{3}$ & 9 & 150 & 7 & 6 & 9 & 16 & 10 & 3 \\
\hline $\mathrm{E}_{3}$ & 13 & 24 & 123 & 21 & 4 & 5 & 15 & 4 \\
\hline $\mathrm{F}_{3}$ & 17 & 18 & 13 & 136 & 11 & 8 & 4 & 3 \\
\hline $\mathrm{G}_{3}$ & 10 & 14 & 20 & 6 & 145 & 7 & 6 & 2 \\
\hline $\mathrm{A}_{3}$ & 10 & 18 & 12 & 16 & 12 & 137 & 2 & 3 \\
\hline $\mathrm{B}_{3}$ & 7 & 2 & 18 & 7 & 13 & 8 & 151 & 4 \\
\hline $\mathrm{C}_{4}$ & 158 & 10 & 7 & 13 & 12 & 4 & 2 & 4 \\
\hline $\mathrm{D}_{4}$ & 5 & 144 & 20 & 10 & 19 & 6 & 5 & 1 \\
\hline $\mathrm{E}_{4}$ & 7 & 10 & 128 & 18 & 17 & 20 & 6 & 4 \\
\hline $\mathrm{F}_{4}$ & 9 & 6 & 5 & 137 & 31 & 17 & 2 & 3 \\
\hline $\mathrm{G}_{4}$ & 10 & 3 & 7 & 4 & 155 & 20 & 10 & 1 \\
\hline $\mathrm{A}_{4}$ & 16 & 2 & 4 & 4 & 10 & 160 & 12 & 2 \\
\hline $\mathrm{B}_{4}$ & 14 & 0 & 10 & 1 & 7 & 6 & 170 & 2 \\
\hline & & & & & & & & 4 \\
\hline
\end{tabular}

Table 2. Frequencies of participants' responses (columns) for each stimulus (rows), independently of the response modality. 


\section{Prpic et al.}

\section{Discussion}

The aim of our work was to verify the existence of constant errors in the identification of pitch classes across consecutive octaves, due to the influence of pitch height. Our findings confirmed that pitch height affects the identification of the pitch class of single tones in expert pianists. Analyzing the direction of the errors, we discovered an intriguing bias. Indeed, when requested to identify the pitch class of target tones between two consecutive octaves, participants showed a general tendency to overestimate pitch classes in the upper octave and to underestimate pitch classes in the lower octave. Therefore, pianists judged pitch classes (e.g., C) in the higher octave (e.g., $\mathrm{C}_{4}=261.6 \mathrm{~Hz}$ ) higher than they actually were, while they judged pitch classes in the lower octave (e.g., $C_{3}=130.8 \mathrm{~Hz}$ ) lower than they actually were, leading respectively to overestimations (e.g., D) and underestimations (e.g., B) in the identification task. Thus, this perceptual bias was probably due to the different pitch height of the pairs of stimuli belonging to the same pitch class. Moreover, this phenomenon does not seem to depend from the response modality, as there were no differences between verbal and motor responses.

In our opinion, participants used both pitch class and pitch height information to solve the task. Undoubtedly, they used pitch class information, since the number of correct responses was quite high (see Table 2). The high accuracy was because the stimuli were very familiar and easy to identify. However, since participants were not AP possessors, they used pitch height information to support their lack in pitch class identification. Indeed, our participants could not rely completely on their pitch class ability and, therefore, their responses were influenced by the pitch height of the stimuli, resulting in overestimation and underestimation of the pitch classes in the upper and lower octaves, respectively. 


\section{Prpic et al.}

These results are consistent with previous findings regarding the influence of pitch height on pitch class identification (Bachem, 1937; Baird, 1917; Miyazaki, 1989; Rakowski \& Morawska-Bungeler, 1987; Ward, 1963). However, in contrast to previous studies, our findings highlight some specific features of this phenomenon, revealing a systematic bias in the direction of the judgments. We call this effect "pitch class polarization", since judgments tends to go towards opposite directions: pitch classes are underestimated in the lower octave and overestimated in the upper octave.

The pitch class polarization is an intriguing phenomenon for music theory. Indeed, one of the main assumptions of music theory is octave equivalence, that is, tones standing one or more octave apart are considered musically identical. However, the analysis of the interval errors has revealed that pitch classes are not identified as identical across octaves, showing a systematic bias. Previous studies revealed contradictory results about octave equivalence, depending on experimental demands. In general all humans can show octave equivalence to a certain degree, however musicians show octave equivalence in more situations than non-musically trained individuals (Allen, 1967; Hoeschele, Weisman, \& Sturdy, 2012; Kallman, 1982; Umemoto, 1990; Thurlow \& Erchul, 1977). Contrary to most of the literature, in which octave equivalence was directly tested, our method indirectly provides some further indication that musicians can violate the octave equivalence principle, since their identification judgments revealed a systematic bias. Thus, our results are in line with those studies which failed to find octave equivalence in musically trained participants (Hoeschele, Weisman \& Sturdy, 2012; Kallman, 1982).

Observing Figure 2, the polarization effect appears to be a phenomenon which consistently affects almost all pitch classes used in this study. However, the significant Octave $x$ Pitch Class interaction and the analyses on single pitch classes suggest that this polarization effect is more evident in some pitch classes $(D, E, F, G)$, compared to others (C, A, B). In our opinion, this result is mainly due to two factors, 


\section{Prpic et al.}

namely the higher familiarity with some pitch classes and the range of stimuli. As for the familiarity with pitch classes, $\mathrm{C}$ and $\mathrm{A}$ are most likely more familiar compared to other pitch classes, because $\mathrm{C}$ is the tonic and the head tone of the C-major scale, while $\mathrm{A}$ is a frequently used pitch class, as the middle A (i.e., $A_{4} 440 \mathrm{~Hz}$ ) serves as tuning standard for musical pitch (Cavanagh, 2009). As for the range of stimuli, $C$ and $B$ are both the extreme points of the $C$ major scale and the lowest $\left(C_{3}\right)$ and the highest $\left(\mathrm{B}_{4}\right)$ stimuli we used in our experiment. Thus, we cannot exclude that participants created a representation of the range of stimuli, and for this reason it is possible that $\mathrm{C}$ and $\mathrm{B}$ (and in particular $\mathrm{C}_{3}$ and $\mathrm{B}_{4}$ ) were easier to identify compared to other stimuli. Indeed, the number of hits for $\mathrm{C}_{3}$ and $\mathrm{B}_{4}$ were the highest (see Table 2), revealing a sort of ceiling effect at the extreme points of the range of stimuli.

The role of the range of stimuli and, in general, the pitch class polarization effect should be further investigated in future studies. In particular, it would be interesting to replicate the polarization effect using different sets of stimuli, such as the chromatic scale, and to ascertain the existence of this bias also in non-consecutive octaves and across multiple octaves. In the latter case, it could be particularly interesting to investigate this effect across an odd number of octaves (e.g., three octaves), to verify the identification errors in the middle octave. It would be reasonable to expect a non-biased identification in the middle octave and, respectively, an underestimation and an overestimation in the lower and upper octaves. Moreover, we suppose that the pitch class polarization effect would grow as the distance between the octaves increases.

In conclusion, we would like to highlight a reflection on a methodological issue. In our opinion, an important point of this study was its focus on the error analysis of the pitch class identification trials. Indeed, through the analysis of the direction and of the amount of errors (Levitin 1994), we have been able to show how the judgment of expert pianists is affected by pitch height, revealing a pitch class 


\section{Prpic et al.}

polarization effect. Due to the particular nature of this effect (i.e., change of error direction depending on the octave) it would have been undetectable by using a dichotomous response paradigm (e.g. correct/incorrect), without analyzing the error direction. 
Prpic et al.

\section{References}

Allen, D. (1967). Octave discriminability of musical and non-musical subjects. Psychonomic Science, 7(12), 421-422.

Babbitt, M. (1960). Twelve-tone invariants as compositional determinants. Musical Quarterly, 246-259.

Babbitt, M. (1965, October). The structure and function of musical theory: I. In College Music Symposium (pp. 49-60). The College Music Society.

Bachem, A. (1937). Various types of absolute pitch. Journal of the Acoustical Society of America, 9,146151.

Bachem, A. (1955). Absolute pitch. Journal of the Acoustical Society of America, 27,1180-1185.

Baird, J. W (1917). Memory for absolute pitch. In E. C. Sanford (Ed.), Studies in psychology, Titchener commemorative volume (pp. 43-78). Worcester, MA: Wilson.

Burns, E. M. (1999). Intervals, scales, and tuning. In D. Deutsch (Ed.), The psychology of music (2nd ed., pp. 215-264). San Diego, CA: Academic Press.

Burns, E. M., \& Ward, W. D. (1978). Categorical perception-phenomenon or epiphenomenon: Evidence from experiments in the perception of melodic musical intervals. The Journal of the Acoustical Society of America, 63(2), 456-468.

Cavanagh, L. (2009). A brief history of the establishment of international standard pitch a $=440 \mathrm{~Hz}$. WAM: Webzine about Audio and Music, 4. 


\section{Prpic et al.}

Crickmore, L. (2003). A re-valuation of the ancient science of harmonics. Psychology of Music, 31(4), 391-403.

Deutsch, D. (1972). Octave generalization and tune recognition. Perception \& Psychophysics, 11(6), 411-412.

Deutsch, D., Dooley, K., \& Henthorn, T. (2008). Pitch circularity from tones comprising full harmonic series. The Journal of the Acoustical Society of America, 124(1), 589-597.

Deutsch, D. (2013). Absolute Pitch. In D. Deutsch (Ed.), The Psychology of Music (Third Edition) (pp. 141-182). London: Academic Press.

Forte, A. (1973). The structure of atonal music (Vol. 304). Yale University Press.

Hoeschele, M., Weisman, R. G., \& Sturdy, C. B. (2012). Pitch chroma discrimination, generalization, and transfer tests of octave equivalence in humans. Attention, Perception, \& Psychophysics, 74(8), $1742-1760$.

Kallman, H. J. (1982). Octave equivalence as measured by similarity ratings. Perception \& Psychophysics, 32(1), 37-49.

Levitin, D. J. (1994). Absolute memory for musical pitch: evidence from production of learned melodies. Perception and Psychophysics, 56(4), 414-423.

Levitin, D. J. (2002). Memory for musical attributes. Foundations of cognitive psychology: Core readings. 


\section{Prpic et al.}

Levitin, D. J., \& Rogers, S. E. (2005). Absolute pitch: perception, coding, and controversies. Trends in cognitive sciences, 9(1), 26-33.

Lockhead, G. R., \& Byrd, R. (1981). Practically perfect pitch. Journal of the Acoustical Society of America, 70, 387-389.

Miyazaki, K. (1988). Musical pitch identification by absolute pitch possessors. Perception \& psychophysics, 44(6), 501-512.

Miyazaki, K. (1989). Absolute pitch identification: Effects of timbre and pitch region. Music Perception, Vol. 7, No. 1, 1-14.

Owen, H. (2000). Music Theory Resource Book. Oxford University Press.

Oxenham A. J. (2013). The perception of musical tones. In D. Deutsch (Ed.), The Psychology of Music (Third Edition) (pp. 1-33). London: Academic Press.

Profita, J., \& Bidder, T. G. (1988). Perfect pitch. American Journal of Medical Genetics, 29, 763-771.

Rakowski, A., \& Morawska-Bungeler, M. (1987). In search for the criteria of absolute pitch. Archives of Acoustics, 12, 75-87.

Russo, F. A., Cuddy, L. L., Galembo, A., \& Thompson, W. F. (2006). Sensitivity to tonality across the pitch range. Perception, 36(5), 781-790.

Shepard, R. N. (1982). Geometrical approximations to the structure of musical pitch. Psychological review, 89(4), 305. 


\section{Prpic et al.}

Takeuchi, A. H., \& Hulse, S. H. (1991). Absolute-pitch judgments of black and white-key pitches. Music Perception, 27-46.

Takeuchi, A. H. \& Hulse, S. H. (1993). Absolute pitch. Psychological Bulletin, 113, 345-36.

Thurlow, W. R., \& Erchul, W. P. (1977). Judged similarity in pitch of octave multiples. Perception \& Psychophysics, 22(2), 177-182.

Umemoto, T. (1990). The psychological structure of music. Music perception, 115-127.

Vanzella, P., \& Schellenberg, E. G. (2010). Absolute pitch: Effects of timbre on note-naming ability. PloS one, 5(11), e15449.

Vos, P. G., \& Troost, J. M. (1989). Ascending and descending melodic intervals: Statistical findings and their perceptual relevance. Music Perception, 383-396.

Ward, W. D. (1963). Absolute pitch: Part II. Sound, 2, 33-41.

Ward, W. D., \& Burns, E. M. (1999). Absolute pitch. The psychology of music, 2, 265-298.

Weisman, R. G., Balkwill, L. L., Hoeschele, M., Moscicki, M. K., Bloomfield, L. L., \& Sturdy, C. B. (2010). Absolute pitch in boreal chickadees and humans: exceptions that test a phylogenetic rule. Learning and Motivation, 41(3), 156-173.

Wynn, V. T. (1993). Accuracy and consistency of absolute pitch. PERCEPTION-LONDON-, 22, 113-113.

Zatorre, R., \& Beckett, C. (1989). Multiple coding strategies in the retention of musical tones by possessors of absolute pitch. Memory \& Cognition, 17, 582-589. 